\title{
Choosing a lifting posture: squat, semi-squat or stoop
}

\begin{abstract}
The lifting of low-lying objects is a known risk factor lower back injuries. Choosing the most appropriate lifting posture is an important part of safe lifting training. However, there is considerable debate as to which is the best technique to employ when lifting low-lying objects off the floor. Selecting the correct lifting posture that matches the task requirement is a critical injury mitigation strategy. Of the different types of lifting techniques, the squat lift is among the most common types of lifting techniques used to lift low-lying objects. However, other lifting techniques such as the scoop and semi-squat lift may offer more flexibility. The purpose of this mini-review is to compare and contrast the benefits of three different lifting techniques the squat lift, the stoop and semi-squat lift.
\end{abstract}

Volume 2 Issue 2 - 2017

Luke Del Vecchio

Department of Medical and Applied Sciences, Central

Queensland University, Australia

Correspondence: Luke Del Vecchio, Department of Medical and Applied Sciences, Central Queensland University,Australia, Email I.delvecchio@cqu.edu.au

Received: June 13, 2017 | Published: July 17, 2017

\section{Introduction}

Manual Handling involves the lifting, carrying, pushing and pulling of materials and objects. ${ }^{1}$ Training in safe handling techniques is a recommended way to reduce risk factors associated with manual handling within the working environment, particularly the lifting of low-lying objects as this has long been associated with an increased risk of lower back injuries. ${ }^{2}$ However, manual handling training programs have not proven to be successful in reducing musculoskeletal problems in an industrial environment, often attributed to inadequate training methods. ${ }^{2}$ The majority of safe handling training including lifting techniques for low-lying objects is not adaptable to employees, workplaces and the variety of tasks found within an industrial environment. In a broad consensus most governing bodies favor the promotion of sound biomechanical principals associated with manual handling tasks; however, they do not appear to be easily applicable to the wide range and complexity of manual handling situations that occur in an industrial environment. ${ }^{3}$

The injury type and mechanism involved in manual handling need to be fully understood as they relate to various lifting tasks. For example, lower back injuries are among the most common type of injury associated with lifting tasks. ${ }^{2}$ Lower back injuries occur because of the large extensor moments about the joints of the lumbar vertebrae that are produced during lifting by the paravertebral musculature to overcome the flexor moment caused by the weight of the upper body and load. ${ }^{4}$ These high forces also result in large compressive and shear forces acting between each pair of vertebra. Studies from cadaveric spines $^{4,5}$ demonstrate when the lumbar spine is in a posture of extreme flexion, the mechanism of failure due to a single compressive load is a failure of the endplates of the vertebral bodies and the underlying trabeculae as the nucleus pulpous bulges upward and downward. ${ }^{4,5}$ The extent of compressive forces experienced during a single lift is improbable to cause endplate failure and injury is more likely to be cumulative. ${ }^{4}$ Thus, lifting with a flexed spine can substantially increase the risk of lower back injury. Moreover, cumulative damage to vertebral endplates also occurs in some ways. Furthermore, microdamage to vertebral endplates is likely during heavy lifting and injury may arise if the micro-damage accumulates more rapidly than can be repaired. ${ }^{4,5}$ Finally, repeated compressive loading can also reduce the failure tolerance of the tissues, resulting in injury if the repeated loading continues ${ }^{4,5}$ (Figure 1).

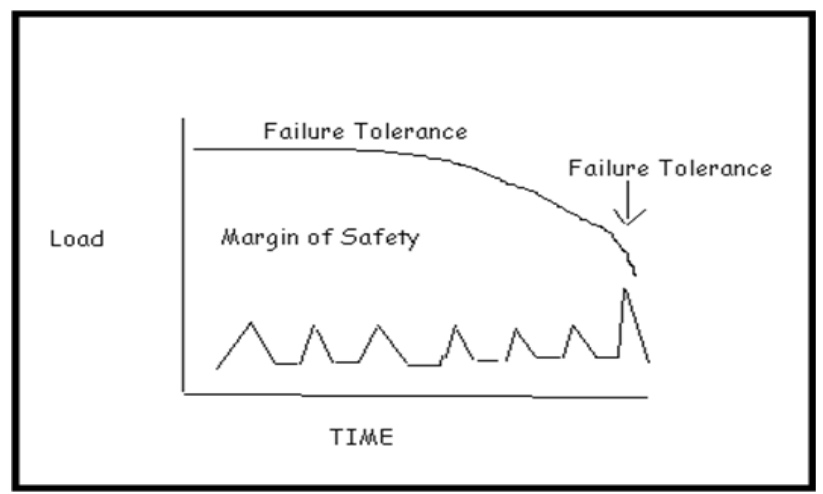

Figure I Repeated sub failure loads lead to tissue fatigue, reducing the failure tolerance of the low the back tissues; leading to failure on the $\mathrm{N}^{\text {th }}$ repetition of the sub-maximal load. ${ }^{5}$

To determine which manual handling tasks may predispose the spine to greatest the greatest injury risk, researchers have undertaken investigations of lower back compression loading during general manual handling duties. ${ }^{5,6}$ For example, researchers ${ }^{6}$ measured low back loading during a variety of handling tasks, and reported three distinct compression loading classifications:

I. Class 1: high compression loading ( mean load: 4,283 Newton's)

II. Class 2: Moderate compression loading (mean load: 3074 Newton's)

III. Class 3: Low compression loading (mean load: 1907 Newton's)

The highest lower back loading (compression) were found in tasks that involved vertical lifting tasks. ${ }^{6}$ Research has reported approximately 7,000 Newton's of compression begins to cause damage in fragile spines. ${ }^{5}$ In contrast, the tolerance of the lumbar spine in an average healthy young man probably approaches 9,000 Newton's. ${ }^{7}$ To 
provide some context, consider the effect is lifting a $27 \mathrm{~kg}$ item held in the hands using a squat style lift would have on the spine, lifting this load will impose approximately 7,000 Newton's of compression to the spine. ${ }^{5}$ The Revised N.I.O.S.H equation manual handling tasks in class 2 and three should be safe to carry out for most workers, as the proposed safety limit of 3,400 Newton's should protect $99 \%$ of male employees and $75 \%$ of working women. ${ }^{8}$ Further suggestions have also been made regarding the consideration that age and gender play, and that these are factors that influence a person's physical capacity (including spinal strength). Proposals ${ }^{9}$ have been made to include age and gender-specific limits based on these biomechanical findings. For example, proposed limits for women should range from 4,400 Newton's at the age of 20,3,200 Newton's at the age of 40 and finally to 1,800 Newton's at the age of 60 or more. ${ }^{9}$ According to Mc Gill, ${ }^{5}$ The spine is at much greater risk of sustaining shear injury than compressive injury (>1000 Newton tolerance for shear Vs. 3000 Newton tolerance for compression).

Within the literature $\mathrm{e}^{1-3,10,11}$ there is considerable debate as to which is the best technique to employ when lifting low-lying objects off the floor; "Stoop" or "Squat." The most commonly advised lifting technique is the "Squat technique or leg lift," which is characterized by a starting position of deep knee flexion with the trunk close to erect, quantitatively this can define by knee flexion of $45^{\circ}$ and trunk flexion less than $30^{\circ}$ when lifting from the floor level..$^{2,10}$ The "Stoop" technique involves spinal flexion, defined as raising a low lying object with a knee flexion angle greater than $135^{\circ}$ with trunk flexion around $90^{\circ} .2,12$

Interestingly, a third technique modification between the stoop lift and squat lift known as the "semi-squat lift" may offer an alternative lifting posture, for the lifting of low-lying objects. The semi-squat lift uses a posture mid-way between the squat and stoops lifts. ${ }^{13}$ Quantitatively the Semi-Squat lift demonstrates knee flexion angles around $90^{\circ}$ and trunk flexion around $45^{\circ}$ for most individual lifting an object from the floor leve $1^{10}$ (Figure 2).

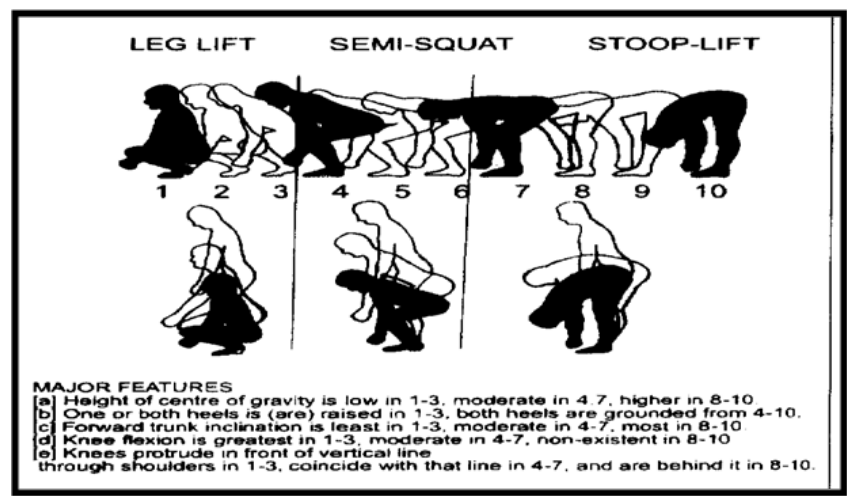

Figure 2 Shows the continuum of lifting starting positions and related lifting movements.

\section{Squat technique}

Antidotal surveys of manual handling professionals demonstrate that the squat lift is widely regarded as the 'correct technique' for lifting low-lying objects. ${ }^{10}$ Historic references to squat type lifting were noted in 1945 when James Cyriax (Assistant Medical Officer, Physiotherapy Department, St. Thomas's Hospital) in a letter published in the Lancet, October 6, 1945) suggested that patients liable to lumbago (low back pain) must avoid heavy work involving trunk-flexion. They must learn to kneel and squat instead of bending forwards; it is full flexion said Cyriax that encourages the onset of Lumbago. ${ }^{4}$ This passage historically was one of the earliest occurrences of a recommendation regarding altering lifting technique using a squat technique to avoid back injury.

Interestingly, Cyrix's comments regarding the benefits of avoiding heavy work involving trunk flexion were restricted to such genetically predisposed persons, somewhere along the line it became an article of faith that lifting should be carried out from a full squat posture, despite many researchers noting that the recommendation was unjustified. Is there conclusive evidence to support the use of a squat style lift, considering most workplace training manuals and media promote this technique? Published studies ${ }^{[2,13]}$ comparing squat lifting to stoop lifting have had their methodologies questioned as to whether during these studies a true squat technique used or was an indiscriminate version of the squat technique used thus invalidating the comparison. ${ }^{10}$

\section{Physiological evidence}

It has been suggested that the traditionally recommended straight back, bent knee (squat) method of lifting is slower and physiologically more demanding resulting in a higher total oxygen consumption for squat lifting, although recently it has been shown that squat lifting has a higher capacity. ${ }^{10}$ Additional research ${ }^{14}$ compared ten male forestry workers' oxygen consumption and heart rates for a task involving the squat and stoop life. The researchers ${ }^{14}$ reported, maximum oxygen consumption for stoop lifting was $14.3 \%$ less than for squat lifting. Also reported in this study were maximum ventilation capacities, which were $18.7 \%$ less than squat lifting and heart rate maximum capacities for stoop lifting were reported to be $6.5 \%$ less than squat lifting. ${ }^{14}$ Further research ${ }^{14}$ has also demonstrated that the squat method requires higher oxygen consumption and higher inspiratory ventilation volumes when compared against stoop lifting, and was subjectively rated more tiring than a stoop lift. ${ }^{14}$ Thus, these results indicate that workers who select the squat technique for a continuous lifting task would need moderately good levels of aerobic capacity to be able to sustain this technique.

\section{Biomechanical evidence}

The squat lift utilizes a specific strategy of avoiding spinal flexion, and this has quite dramatic effects on shear loading of the intervertebral column and resultant injury risk. ${ }^{5}$ The dominant direction of the erector spinae (longissimus thoracic and iliocostalis lumbar muscles) when the lumbar spine remains in a neutral lordosis causes these muscles to produce a posterior shear force on the superior vertebra thus stabilizing the vertebrae from aberrant motion. ${ }^{5}$ If the lifter adopts a flexed spine when lifting (stoop lift) the interspinous ligament becomes strained and generates forces with the opposite obliquity, therefore imposing anterior shear force on the superior vertebra and destabilizing the vertebra making it more susceptible to injury. ${ }^{5} \mathrm{As} \mathrm{McGill}^{5}$ reports, when the interspinous ligament is recruited in this manner the resultant shear force levels seem likely to exceed 1000 Newton's, such large forces predispose the worker/lifter to injury. On the contrary, when the lifter adopts a squat lifting posture maintaining the neutral lordotic curves the erector spinae and other extensor musculature are responsible for opposing this anterior shear with a posterior shear force that supports the anterior shearing action of gravity on the upper body and the load held in the hands. ${ }^{5}$ The joint 
shear forces are reduced to 200 Newton's, allowing the muscles to support the moment in a more neutral posture rather than being fully flexed with ligaments supporting the moment, and hence significantly reduces the shear loading to much safer levels., 4 Shear tolerances of the spine have been postulated to be in the neighborhood of 20002800 Newton's. ${ }^{5,15}$ Research ${ }^{16}$ has further confirmed that estimates of shear forces for stoop lifting to be 180 percent greater than for squat lifting. Taken together, these data suggest squat lifting imposes much lower shear forces on the spine than stoop lifting.

Passive tissue stress also has been found to be lower in a squat lift as opposed to the stoop lift. ${ }^{17}$ Stresses on discs and ligaments for men and women performing squat and stoop lifting and expressing their results as a percentage of elastic limit for those tissues found stoop lifting to result in around $75 \%$ more stress on the passive tissues. ${ }^{17}$ Compressive strength and disc load have been carefully studied on cadaveric spines; the optimum end plate compressive strength and equal compressive and tensile forces in the annulus fibrosis at $75 \%$ of maximum lumbar flexion which is the ranges of motion seen in a squat lift. ${ }^{18}$ The optimum range for resisting compression, therefore, appeared to be $0-75 \%$ flexion ${ }^{[19]}$. The consensus from these studies indicate that a fully flexed spine, is weaker than one that is less flexed, as seen in both the modified squat lift and squat lift.18

Peak Lumbar moment $(\mathrm{Nm})$ may be lower in the squat lift as opposed to the stoop lift; data ${ }^{16}$ from 15 men lifting weights from $6-32 \mathrm{kgs}$ demonstrated a $5 \%$ lower peak lumbar moment in the squat lift against the stoop lift. Disc herniations are another common lifting injury, which is strongly correlated to repeated bouts of spinal flexion. ${ }^{19}$ This type of spinal motion is commonly seen in workers who perform long bouts of lifting tasks using a stoop technique, only a very modest amount of spine compression force (800-1,000 Newton's) is needed to cause a disc herniation's during repeated bouts of spinal flexion. ${ }^{19}$ Researchers ${ }^{19}$ were able to accurately predict how many cycles of full spinal flexion would cause the disc nucleus material to travel posteriorly through the annulus thus causing herniation. The researchers observed 18,000-25,000 cycles of flexion with low levels of spinal compression (1000 Newton's) or 5,000 cycles if there were larger compression loads ( 3,000 Newton's) resulted in injury to the spine. ${ }^{19}$ The results of this study demonstrate that repeated bouts of spinal flexion as seen in a stoop lift even in the absence of moderate loads may increase the risk of lumbar intervertebral disc injury. ${ }^{5}$ Using the squat lift enables more stress to be placed on the muscles, and less on the ligaments, nerve and disc structures providing the squat is carried out with $<75$ degree's of spinal flexion. Taken together, this data suggests, using the squat lift may be a preferable to the stoop lift, for preventing lower back injuries.

\section{Squat Summary}

Over the past years there has been modest evidence at best to support the use of the squat lift for low-lying objects, Evidence presented here to support the rationale of using a squat lift includes:

i. Much lower lumbar shear forces.

ii. Lower lumbar compressive forces.

iii. Higher tolerance of the joint to withstand compressive forces.

iv. Less lumbar passive tissue stress.

\section{v. Lower peak lumbar moment.}

vi. Eliminate the risk of disc herniations.

vii. Eliminate the risk of strain to the interspinous ligament complex.

viii. Greater use of spinal musculature; thus maximizing shear support

ix. Spares passive tissues, thus preferable to injure muscle rather than ligaments.

x. People instinctively know to perform lifting this way.

\section{Semi-squat technique}

The stoop and Squat lifts have been well described in the literature; however, less attention has been given to the semi-squat Lift. The semi-squat lift combines elements of the stoop and squat lifting. ${ }^{10}$ To overcome limitations of previous research conducted on lifting techniques ${ }^{19}$ a consensus meeting was arranged to sample Australian wide opinion among people with professional interests in manual handling and to set a consensus about appropriate lift training strategies. ${ }^{19}$ The results of this consensus meeting saw $80-90 \%$ of the professional participants agreed that the squat lift was an appropriate lifting technique for lift training. Less than $10 \%$ were prepared to recommend the stoop-lift, while $25 \%$ claimed they were users of the semi-squat approach and claimed to be already using that approach for training purposes. ${ }^{19}$ The traditionally recommended full squat posture is seldom, if ever, spontaneously adopted in the absence of specific instructions; investigations of self-selected lifting technique have demonstrated that postures naturally adopted to lift a low lying object fall between a full squat and a stoop. ${ }^{4}$ To fully define the biomechanics of a semi-squat lift; consideration of the pattern of inter-joint coordination as well as the posture adopted at the start of the movement is required. The posture taken at the beginning of extension influences the pattern of subsequent inter-joint coordination by determining the range of movement available at each joint. ${ }^{4}$ The semi-squat posture most commonly used at the start of extension allows a pattern of inter-joint coordination which appears to be functional. ${ }^{4}$ The semi-squat technique provides a way to avoid some of the difficulties apparent with both stoop and squat lifting; however, the fundamental question remains "is the semi-squat technique biomechanically and physiologically more efficient?"

\section{Physiological evidence}

Results from a study ${ }^{20}$ that compared the oxygen consumption and heart rates between squat lifting and freestyle lifting (the joint angles used in the freestyle lifting technique in this study were closer to the semi-squat technique, knee flexion $>90$ degrees; then a stoop lift. Oxygen consumption and heart rate values were significantly lower in the freestyle lifting technique vs. the squat lift, ${ }^{20}$ also noted was the subject's maximum accepted weight limit (MAW) for freestyle lifting was greater than squat lifting meaning that subjects lift more weight for a similar oxygen consumption. ${ }^{20}$

While the semi-squat lift technique seems to have lower oxygen consumption than the squat lift; data from ${ }^{21}$ found the semi-squat lift to have higher oxygen $(23 \%)$ consumption than stooping. However, the semi-squat lift still demonstrated lower oxygen consumption values in this study as well. External work measurements, therefore, suggest that the energy expenditure for semi-squat lifting should be less than the squat lift, but higher than stoop lifting. 


\section{Biomechanical evidence}

There is confusion amongst the literature as to whether the semisquat lumbar moment is greater than that of the squat lift of stoop lift. ${ }^{10,22}$ One study ${ }^{22}$ found that the semi-squat imposed slightly higher lumbar moment than the squat lift, but went on to comment that the semi-squat, stoop and squat all had lumbar moments within 5\% of each other. Lifting with a semi-squat posture involves about $45^{\circ}$ of lumbar flexion; which is $75 \%$ of the normal range of movement, ${ }^{4}$ suggesting that the passive structures not be significantly stretched during lifting with this posture thus ensuring some safeguarding against a soft tissue injury.

Using a semi-squat lift follows current occupational biomechanical guidelines of avoiding joint extremes, principally it avoids the deep knee flexion angles seen in the squat lift; which can place the knee at a mechanical disadvantage and may present further problems for workers with knee disorders. ${ }^{10}$ Secondly, the squat lift avoids full lumbar flexion seen in the stoop lift, thus keeping tension on the paravertebral musculature and reducing the load on the ligaments, nerves and intervertebral discs. Further examination of the semi-squat lifts demonstrates an advantage over the stoop lift via the different pattern of coordination between the hip and knee that occurs when a stooped posture is adopted at the start of the lifting phase. ${ }^{4}$ The broad range of hip flexion and a small range of knee flexion involved results in the hamstrings being lengthened further than if semisquat posture was adopted. ${ }^{4}$ During a stoop lift, the hamstrings must immediately shorten, because the knee is unable to extend and this counteracts to some extent the strength advantage which might accrue as a consequence of the increased hamstring length (length/tension relationship suggests that muscles are stronger when lengthened and not when shortened rapidly).

It is practically impossible for a substantial number of workers to undertake continuous lifting tasks of low-lying objects - when using the squat method of lifting recommended by the lifting experts. What little evidence that is available does suggest that there may well be some clinical advantages for using the semi-squat lifting technique. Therefore, it has concluded that the traditionally recommended squat style of lifting is slower and physiologically more demanding (Figure $3)$.

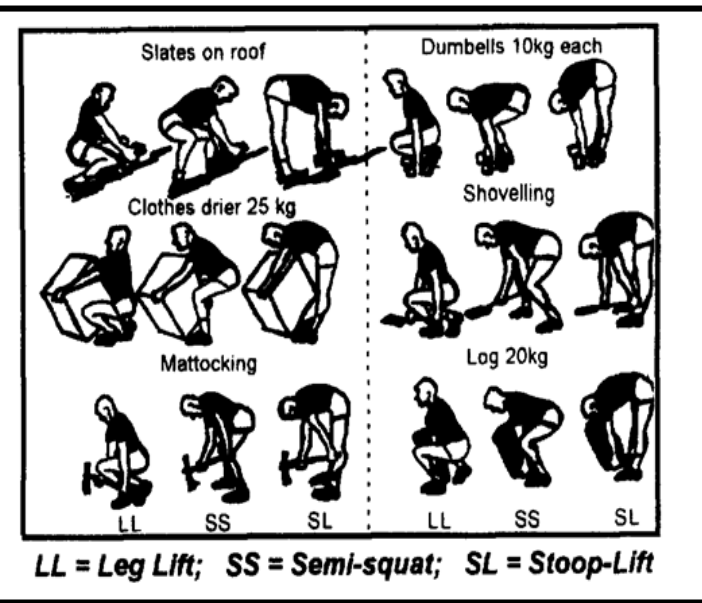

Figure $3 \mathrm{~A}$ comparisons to help the reader make a realistic perception of the differences between the three approaches to lifting. As demonstrated, the greater the lifting demand, the more obvious is the advantages of applying semi-squat principals.

\section{Semi-squat summary}

There is less evidence for or against the Semi-Squat lift than for the squat technique, the evidence in support of using the semi-squat lift includes:

i. Avoids knee and extreme lumbar ranges of motion

ii. Greater Maximum Accepted Weight than for squat and stoop

iii. Less likely to injure lumbar ligaments, intervertebral discs or neural structures

iv. Workers find this to be more of a "Natural" Movement pattern to lift with

v. Avoids high compressive forces on the knee; which may be dangerous for individuals with degenerative knee conditions.

vi. More practical to apply in most occupational environments.

vii. Less fatigue of the knee joint extensors; enabling workers to continue to use this method over greater time periods.

viii. Applies the best mechanics of both squat and stoop and combines them into a more user-friendly approach.

ix. The method has a proven track record in sports like Olympic lifting were most participants utilize a version of the semi-squat, lifting large loads with minimal injuries.

\section{Stoop technique}

Health Professionals and lay people alike commonly understand that the use of a stoop lift is erroneous, and the vast majority of lifting literature advises against it. Interestingly, despite the common perception that stoop lifting can injure the lower back, some researchers support the use of stoop lifting. ${ }^{23,24}$ It is also well recognized that the stoop lift is widely and spontaneously used for "Bent-Over work" such as fruit and vegetable picking, gardening, some housework duties and some light lifting tasks. ${ }^{20}$

\section{Physiological evidence}

Maximum acceptable weight (MAW) is a widely used and recognized manual handling risk assessment criteria. ${ }^{25}$ Researchers ${ }^{26}$ had six young males determine MAW for a broad range of lifting tasks using either a stoop or squat lifting techniques, the participants of this study select a MAW for the stoop lift that was $10-20 \%$ higher than the squat lift. Other researchers ${ }^{10}$ have observed, that young males select a MAW $11.7 \%$ greater than for the squat lift, while the females select a MAW $20 \%$ higher than for squat lifting.

The stoop method of lifting has also been shown by Kumar $\mathrm{S}^{27}$ to require a lower amount of oxygen consumption and Inspiratory ventilation volume than squat lifting. Oxygen consumption was also found to be higher in a squat lift as compared to stoop lifting by Hagen $\mathrm{KB},{ }^{28}$ the researcher's data showed that maximal squat lifting in their subjects utilized a vo2 of $38.7 \mathrm{ml} / \mathrm{kg} / \mathrm{min}$ while the Vo2 for stoop lifting was $32.9 \mathrm{mls} / \mathrm{kg} / \mathrm{min}$. We can conclude from this evidence that a stoop lift requires lower oxygen consumption than a squat lift, which explains why it might be the method of choice used by less fit workers. Secondly lifters using a stoop lift will elect to lift heavier loads.

\section{Biomechanical evidence}

Several studies ${ }^{29,30,31}$ report that lumbar moment for stoop and squat 
to be within $5 \%$ of each other which would come as a surprise to many health professionals. More research ${ }^{32}$ looking at lumbar moments between squat and stoop lifting found lumbar moments were lower $(10 \%)$ for a group of men and women lifting loads between $0-30 \mathrm{kgs}$ using the stoop lift. There has been some early research suggesting that Lumbar compressive forces may be lower in stoop lifting, according to ${ }^{24}$ for most lifting situations stoop lifting results in lower lumbar compressive forces, although this research has now been shown to be inaccurate..$^{27,30}$ More recently ${ }^{16}$ demonstrated that the estimated lumbar compression force in stoop lifting was $10 \%$ lower than that of a squat lift. Importantly a methodological consideration from these studies should be the position that the subjects were asked to lift the load from, as it has been shown ${ }^{12}$ that lifting loads between the feet utilizing the squat technique produce a lower net moment and lumbar compression force. Should the load not be lifted from a position between the feet, the resulting net moments and compression forces will be higher in the squat lift. ${ }^{12}$

Workers will tend to select stoop lifting to avoid fatigue or manage with fatigue. For example, when researchers ${ }^{33}$ examined the effect of lifting frequency on the thigh and lower trunk motion, a significant gradual decrease in thigh motion range was seen for a majority of the subjects during squat lifting at lifting frequency of 20 lifts per minute. The researchers suggested that the changes that occurred in trunk and thigh motion were due to quadriceps muscle strength and this is the limiting factor in repetitive squat lifting. ${ }^{33}$ Further to this, ${ }^{14}$ found that humans may naturally prefer the stoop lift method because of the greater demand a squat lift imposes on the knee extensors. Relatively more mechanical work is required to perform the more commonly recommended squat lift, is also an important factor in explaining why humans naturally prefer the stoop lifting method..$^{14}$

While controversial, some of the evidence presented here supports the claim that lumbar moment and lumbar compression forces may be lower on a stoop lift, although not all researchers agree, stoop lifting appears to more energy efficient and minimizes knee extensor fatigue. The stoop lift appears a more natural lift for most people and ${ }^{34}$ has shown that the knee angles during box handling for expert manual handlers were very similar to the knee flexion angles used by novice lifters. What's more, balance loss during lifting has also been shown to be a risk factor for lower back injury during lifting when using a squat lift. ${ }^{35}$ Health professionals should bear in mind though, compelling research from ${ }^{36}$ that shows prolonged exposure to static postures involving stoop positions (lumbar flexion) will cause the tissues to creep and the stabilizing ligaments do not return to their resting length immediately upon unloading. This temporary loss of stability after the period of sustained extreme lumbar flexion (stooped posture) which may then lead to a higher likelihood that an injury may occur in subsequent lifting or loading tasks. ${ }^{36}$ In the full squat posture, the heels will inevitably lift from the ground, and the knees are in an unstable loose packed posture when maximally flexed. ${ }^{4}$ The possibility of injury may increase due to any unexpected perturbations.

Arguments for health professionals to subscribe to the use of stoop lifting would follow the evidence that most workers will use either a stoop lift or a semi-squat lift and enforce the squat lifting technique would require a change in movement patterns, along with an appropriate working environment (non-confined spaces). Secondly, there could be worse ramifications of trying to change the lifting habits of asymptomatic workers who may lose the protection of a well-practiced and conditioned movement pattern.

\section{Stoop summary}

Surprising none the less, there seems to be reasonable evidence to support the use of stoop lifting for low-lying objects, including:

i. Maximal accepted weights may be higher

ii. Lumbar moments and compression forces may be lower

iii. Energy expenditure, inspiratory ventilation are lower

iv. Quadriceps fatigue is lower

v. More sustainable over long periods of repetitive lifting

vi. More sustainable for lifting tasks that require faster lifting speeds

vii. People naturally stoop.

viii. Trying to change their movement patterns has not been shown to be very effective.

\section{Discussion}

This research review has attempted to summarize the available evidence to support the use of the squat, semi-squat and stoop techniques for lifting low-lying objects. It seems that issue of whether to squat or stoop is more complex. According to Mc Gill ${ }^{5}$ the lumbar spine curvature determines the sharing of the load between muscles and passive tissues, while the reaction moment is a function of the size and position of the load in hands and the position of the center of mass of the upper body.

The squat lift appears to have lower lumbar shear stress and places less stress on the passive tissues of the spine, while stoop lifting seems to be more natural and less fatiguing. The Semi squat lifting may well be a good compromise between the squat and stoop. If it is muscular fatigue that contributes to lifting related injuries, then techniques which reduce muscular fatigue and effort should be advised. There appears to be no single best lifting posture that is appropriate for all situations; rather it may be preferable to provide education and training in general lifting guidelines and efficient biomechanical movement patterns. This way the lifter/ worker can discover individually appropriate postures and movement patterns.

Finally, a case can certainly be made for appropriately designed and delivered strength and conditioning program that targets the development of safe lifting techniques for workers involved in manual handling and lifting tasks and has been suggesting by BurgessLimerick $^{4}$ as a method of reducing the risk of injury to the back. Strengthening the muscles, ligaments, and bones would increase the individuals' capacity to bear the load, lift more confidently and resist injury. While flexibility and range of motion training would allow the lifters to use proper biomechanics while lifting, prevent postural abnormalities and increase their range of the movement at relevant joints required to perform common manual handling tasks. ${ }^{37}$

Based on the current research, general lifting guidelines should include: ${ }^{1,5}$

a. Design work tasks that facilitate variety

b. Wherever possible, remove exposure to manual lifting by providing mechanical aids.

c. If lifting or manual handling tasks must be undertaken, if possible reduce the weight of the objects to be lifted or moved. 
d. Raise the height of the initial height of the load.

e. During lifting, choose a posture to minimize the reaction torque on the low back by keeping the load close to the body.

f. Adopt a posture at the start of the lift which involves a moderate range of motion at the knee, hip and vertebral column.

g. Avoid lifting from a posture of extreme lumbar flexion.

h. Avoid lateral trunk flexion.

i. Avoid lifting heavy objects after sustained periods of lumbar flexion.

j. Avoid twisting and the simultaneous generation of high twisting torques.

k. Ensure that the neutral curves of the spine are maintained as much as possible during lifting tasks.

1. Avoid high acceleration of the load, lift smoothly.

$\mathrm{m}$. Develop the ability to rotate the spine from the hips, thus preserving the neutral curve in the lumbar spine.

n. Pre-stress and stabilize the spine by lightly contracting the stabilizing musculature of the trunk even during the moderate lifting tasks.

o. Practice joint-conserving movement patterns; some workers need to constantly practice motion patterns such as locking the lumbar spine when lifting and rotating the hips.

p. Consider appropriate rest break strategies, workers engaged in dynamic work may be better served with longer more restful breaks.

q. Encourage workers to maintain a reasonable level of fitness.

\section{Acknowledgements}

None.

\section{Conflict of interest}

The author declares no conflict of interest.

\section{References}

1. Stevenson MG. Notes on the Principles of ergonomics. University Printing Service, Australia: University of Sydney; 2006.

2. Vincent SM, Tellier C, Lortie M. Training in handling: an evaluative study Ergonomics. 1989;32(2):191-210.

3. Gagnon M. Ergonomic identification and biomechanical evaluation of workers' strategies and their validation in a training situation: Summary of research. Clin Biomech. 2005;20(6):569-580.

4. Limerick BR. Squat, stoop or something in between. International Journal of Industrial Ergonomics. 2001;31(3):143-148.

5. Gill MSM. Low Back Disorders: evidence-based prevention and rehabilitation. USA: Human Kinetics; 2002.

6. Skotte JH, Essendrop M, Hansen AF, et al. A dynamic 3D biomechanical evaluation of the load on the low back during different patient-handling tasks. Journal of Biomechanics. 2002;35(10):1357-1366.

7. Adams M, Dolan P. Recent advances in lumbar spinal mechanics and thei clinical significance. Clin Biomech. 1995;10(1):3-19.
8. Waters TR, Anderson PV, Garg A, et al. Revised NIOSH equation for the design and evaluation of manual lifting tasks. Ergonomics. 1993;36(7):749-776

9. Jager M, Luttmann A. Assessment of the low back load during manua material handling. Proceedings of the $13^{\text {th }}$ triennial Congress of International Ergonomics Association, Finland: 1997.

10. Straker L. Evidence to support using squat, semi-squat and stoop techniques to lift low-lying objects. Int J Ind Ergonomics. 2003;31(3):149-160.

11. Garg A, Herrin GD. Stoop or Squat: A biomechanical and metabolic Evaluation. AIIE Transactions. 1979;11(4):293-303.

12. Dieen VJH, Hoozemans MJ, Toussaint HM. Stoop or squat: a review of biomechanical studies on lifting technique. Clin Biomech (Bristol, Avon). 1999;14(10):685-696.

13. Welbergen E, Kemper HCG, Knibbe JJ, et al. Efficiency and effectiveness of stoop and squat lifting at different frequencies. Ergonomics. 1991;34(5):613-624

14. Giat Y, Pike N. Mechanical and electromyography comparison between the stoop and squat lifting methods. Journal of Safety Research. 1992;23:95105 .

15. Cripton P, Bellmen U, Visiting H Begeman PC. The response of the lumbar spine due to shear loading. Injury Prevention through biomechanics. 1995. $11 \mathrm{p}$

16. Povin JR, Gill MSM, Norman RW. Trunk muscle and lumbar ligamen contributions to dynamic lifts with varying degrees of trunk flexion. Spine. 1991;16(9):1099-1107.

17. Dolan P, Mannion AF, Adams MA. Passive tissues help the back muscles to generate extensor moments during lifting. J Biomech. 1994;27(8):10771085 .

18. Adams MA, Nally MDS, Chinn $\mathrm{H}$, et al. The clinical biomechanics award paper 1993 Posture and the compressive strength of the lumbar spine. Clin Biomech (Bristol, Avon). 1994;9(1):5-14.

19. Callaghan JP, Gill MSM. Intervertebral disc herniation: Studies on a porcine model exposed to highly repetitive flexion/extension motion with compressive force. Clin Biomech (Bristol, Avon). 2001;16(1):28-37.

20. Sedgwick AW, Gormley JT. Training in lifting: An unresolved ergonomic issue? Appl Ergon. 1998;29(5):395-398.

21. Kumar S, Garand D. Static and dynamic lifting strength at differen reach distances in symmetrical and asymmetrical planes. Ergonomics. 1992;35(7-8):861-880.

22. Mittal M, Malik SL. Biomechanical evaluation of lift postures in adult koli female laborers. Ergonomics. 1991;34(1):103-108.

23. Garg A, Herrin GD. Stoop or squat: a biomechanical and metabolic evaluation. AIIE Transactions. 1979;11(4):293-302.

24. Park KS, Chaffin BD. A biomechanical evaluation of two methods of manual load lifting. AIIE Transactions. 1974;6(1):105-113

25. Straker LM. A critical appraisal of manual handling risk assessment literature. Australia: International Ergonomics Association Press; 1997. $131 \mathrm{p}$.

26. Garg A, Saxena U. Physiological stresses in warehouse operations with a particular reference to lifting technique and gender. American Industrial Hygiene Association Journal. 1985;46(10):53-59.

27. Kumar S. The Physiological cost of three different methods of lifting in sagittal and lateral planes. Ergonomics. 1984;27(4):425-433.

28. Hagen KB, Hallen J, Ringdahl KH. Physiological and subjective responses to maximal repetitive lifting employing stoop and squat technique. 
European Journal of Applied Physiology and Occupational Physiology. 1993;67(4):291-297.

29. Looze DMP, Kingma I, Thunnissen W, et al. The evaluation of a practical biomechanical model estimating lumbar moments in occupational activities. Ergonomics. 1994;37(9):1495-1502.

30. Ekholm J, Arborelius UP, Nemeth G. The load on the lumbar-sacral joint and trunk muscle activity during lifting. Ergonomics. 1982;25(2):145-161.

31. Hagen KB, Ringdahl K. Influence of lifting technique on perceptual and cardiovascular responses to submaximal repetitive lifting. Eur J Appl Physiol Occup Physiol. 1994;68(6):477-482.

32. Dolan P, Mannion AF, Adams MA. Passive tissues help the back generate extensor moments during lifting. J Biomech. 1994;27(8):1077-1085.

33. Hagen K, Sorhagen O, Ringdahl HK. Influence of weight and frequency on the thigh and lower trunk motion during repetitive lifting employing stoop and squat techniques. Clin Biomech (Bristol, Avon). 1999;10(3):122-127.
34. Authier M, Lortie M, Gagnon M. Manual Handling Techniques: comparing novices and experts. International Journal of Industrial Ergonomics. 1996;17(5):419-429.

35. ToussaintHM, Commissaris DA, BeekPJ.Anticipatory postural adjustments in the back and leg lift. Med Sci Sports Exerc. 1994;29(9):1216-1224.

36. Gill MSM. The biomechanics of low back injury: implications for current practice in industry and the clinic. J of Biomech. 1997;30(5):465-475.

37. Garg A, Herrin GD. Effects of lifting frequency and technique on physical fatigue with a particular reference to psychophysical methodology and metabolic rate. American Industrial Hygiene Association Journal. 1985;46(2):53-59. 